ANNALES

POLONICI MATHEMATICI

$55(1991)$

\title{
Some criteria for the injectivity of holomorphic mappings
}

\author{
by Staniseaw Spodzieja (Łódź)
}

Abstract. We prove some criteria for the injectivity of holomorphic mappings.

Let $K \subset \mathbb{C}^{n}$ be a bounded and closed domain such that

(1) no closed proper subset of $\partial K$ disconnects $\mathbb{C}^{n}$.

THEOREM 1. If a mapping $f: K \rightarrow \mathbb{C}^{n}$ is continuous, the restriction $f_{\mid \operatorname{Int} K}$ is holomorphic and $f_{\mid \partial K}$ is injective, then $f$ is injective.

Proof. The proof will be carried out in three steps:

1. $f_{\mid \operatorname{Int} K}: \operatorname{Int} K \rightarrow \mathbb{C}^{n}$ is an open mapping. By the assumption, for any $y \in \mathbb{C}^{n}, f^{-1}(y) \cap \partial K$ has at most one point. Consequently, from the Remmert-Stein theorem on removable singularities, $f$ has isolated fibres. So, by Remmert's theorem on open mappings, $f_{\mid}$Int $K$ is an open mapping.

2. $f(\partial K) \cap f(\operatorname{Int} K)=\emptyset$. It is known (see [1], Cor. in Sec. 12, p. 248) that if $A, B \subset \mathbb{R}^{m}$ are compact and homeomorphic, and $A$ disconnects $R^{m}$, then so does $B$. Hence and from (1), $f(\partial K)$ disconnects $\mathbb{C}^{n}$, but no closed proper subset of $f(\partial K)$ does. Since $f(\operatorname{Int} K)$ is open, $f(\operatorname{Int} K) \subset$ Int $f(K)$. Consequently, $\partial f(K) \subset f(\partial K)$. Since $\partial f(K)$ disconnects $\mathbb{C}^{n}$, we get $\partial f(K)=f(\partial K)$, and so $f(\partial K) \cap f(\operatorname{Int} K)=\emptyset$.

3. $f$ is injective. Let $V=\{(x, y) \in K \times K: f(x)=f(y)\}$. Then each irreducible component of $V \cap \operatorname{Int}(K \times K)$ has a positive dimension. Define

$$
g_{i}: V \ni\left(x_{1}, \ldots, x_{n}, y_{1}, \ldots, y_{n}\right) \mapsto x_{i}-y_{i} \in \mathbb{C}, \quad i=1, \ldots, n .
$$

By the maximum principle for holomorphic functions on analytic sets, there exist $\left(x_{0}^{i}, y_{0}^{i}\right) \in \partial(K \times K) \cap V, i=1, \ldots, n$, such that

$$
\left|g_{i}\left(x_{0}^{i}, y_{0}^{i}\right)\right|=\max _{(x, y) \in V}\left|g_{i}(x, y)\right|, \quad i=1, \ldots, n .
$$

1991 Mathematics Subject Classification: Primary 32H99. 
From the definition of $V$ we have $f\left(x_{0}^{i}\right)=f\left(y_{0}^{i}\right)$, thus, by step $2, x_{0}^{i}, y_{0}^{i} \in$ $\partial K$. Hence and from the injectivity of $f_{\mid \partial K}$ we have $x_{0}^{i}=y_{0}^{i}$, that is, $g_{i}\left(x_{0}^{i}, y_{0}^{i}\right)=0$, and thus $g_{i} \equiv 0$ for $i=1, \ldots, n$. Hence $V=\{(x, x): x \in K\}$, therefore $f$ is injective.

The proof is complete.

R e m a r k. In the case $n=1$, this theorem is known (see [2], $§ 11$, Ch. IV, p. 209).

We shall now give another criterion in which we weaken the assumption on the boundary of the domain at the cost of strengthening the assumption on the mapping.

Let $D \subset \mathbb{C}^{n}$ be a bounded and closed domain with connected boundary.

Theorem 2. If $f: D \rightarrow \mathbb{C}^{n}$ is a continuous mapping, $f_{\mid} \operatorname{Int} D$ is holomorphic, $f_{\mid \partial D}$ is injective, and

(2) each $x \in \partial D$ has a neighbourhood $U \subset \mathbb{C}$ such that $f_{\mid U \cap D}$ is injective, then $f$ is injective.

Proof. The proof will be carried out in three steps:

1. $f_{\mid \operatorname{Int} D}: \operatorname{Int} D \rightarrow \mathbb{C}^{n}$ is an open mapping. This is proved in the same way as step 1 in the proof of Theorem 1 .

2. $f(\partial D) \cap f(\operatorname{Int} D)=\emptyset$. Assume to the contrary that $f(\partial D) \cap$ $f(\operatorname{Int} D) \neq \emptyset$. By step $1, f(\partial D) \cap f(\operatorname{Int} D)$ is open in $f(\partial D)$. Take any sequence $y_{n} \in f(\partial D) \cap f(\operatorname{Int} D)$ such that $\lim y_{n}=y_{0}$. Then there exist sequences $z_{n} \in \partial D, x_{n} \in \operatorname{Int} D$ such that $f\left(z_{n}\right)=y_{n}, f\left(x_{n}\right)=y_{n}$. Passing to subsequences if necessary, we may assume that $\lim x_{n}=x_{0}$, $\lim z_{n}=z_{0}$. From (2) we have $z_{0} \neq x_{0}$. So, from the injectivity of $f_{\mid \partial D}$ we get $z_{0} \in \partial D, x_{0} \in \operatorname{Int} D$. In consequence, $y_{0} \in f(\partial D) \cap f(\operatorname{Int} D)$. Thus $f(\partial D) \cap f(\operatorname{Int} D)$ is closed in $f(\partial D)$, that is, by the connectedness of $\partial D$, $f(\partial D)=f(\partial D) \cap f(\operatorname{Int} D)$. To sum up, $f(D)=f(\operatorname{Int} D)$, which is impossible because $f(D)$ is compact and $f(\operatorname{Int} D)$ open.

3. $f$ is injective. This is proved in the same way as step 3 in the proof of Theorem 1.

The proof is complete.

COROllary. If $f: D \rightarrow \mathbb{C}^{n}$ is a holomorphic mapping such that $f_{\mid \partial D}$ is injective and the Jacobian of $f$ does not vanish anywhere in $D$, then $f$ is injective.

We shall now give an example illustrating the fact that the assumptions (1) in Theorem 1 and (2) in Theorem 2 cannot be omitted.

EXAmple 1. Let $B=\exp (\{z \in \mathbb{C}:|z|<\pi\})$. Take a homography $h$ such that $h(\pi i)=2 i, h(-\pi i)=-2 i, h(-\pi)=-1$, and a function $f: B \rightarrow \mathbb{C}$ 
defined by

$$
f(z)= \begin{cases}{[h(\log z)]^{2},} & z \in B \backslash\{-1\} \\ -4, & z=-1\end{cases}
$$

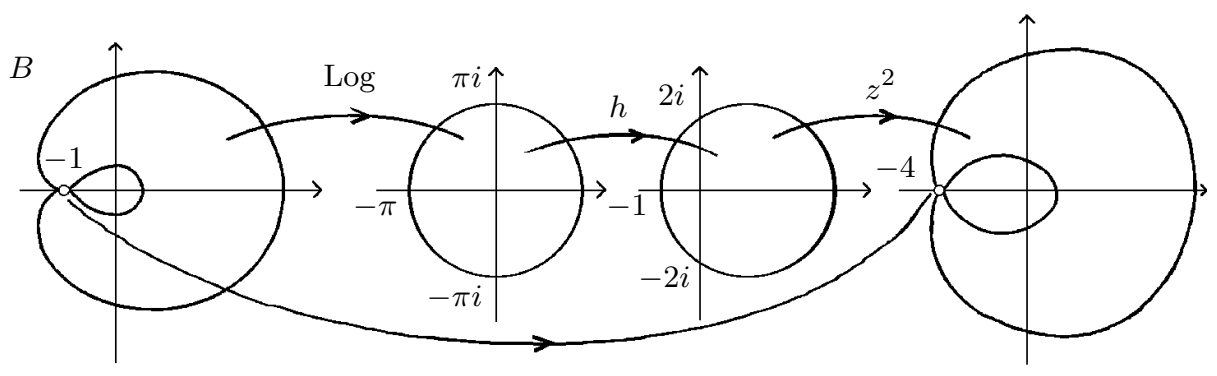

Then $f$ and $B$ have the following properties:

1) $f$ is injective on $\partial B$,

2) $\partial B$ does not satisfy (1),

3) $f$ does not satisfy (2) at the point -1 ,

4) $f$ is not injective in $B$.

It is easy to show, using the Osgood-Brown theorem, that we need not assume the connectedness of the boundary of the domain in Theorem 2 for $n \geq 2$. In the case $n=1$, this assumption is essential, which is shown by the following example.

ExAmple 2. Let $D=\{z \in \mathbb{C}: 1 / 5 \leq|z| \leq 4\}$ and $f: D \rightarrow \mathbb{C}$, $f(z)=z+1 / z$. It is easy to see that $f_{\mid \partial D}$ is injective. Since $f^{\prime}(z)=0$ for $z=1$ and $z=-1$, condition (2) in Theorem 2 is also satisfied. But $f(3)=f(1 / 3)$, thus $f$ is not injective.

\section{References}

[1] K. Borsuk, Über Schnitte der n-dimensionalen Euklidischen Räume, Math. Ann. 106 (1932), 239-248.

[2] S. Saks and A. Zygmund, Analytic Functions, PWN, Warszawa 1965.

INSTITUTE OF MATHEMATICS

ŁÓDŹ UNIVERSITY

BANACHA 22

90-238 ŁÓDŹ, POLAND 